\title{
Research Paper: The Recovery Curve of Soleus and Gastrocnemius H-Reflex After Applying Tripolar Electrical Stimulation on the Anesthetized Skin of Vertebral Column: An Emphasis on the Early and Secondary Facilitation Phases

\author{
Yasamin Khoshamooz ${ }^{1}$, Giti Torkaman $^{2 *}$, Alireza Sarmadi
}

1. MSc., Department of Physiotherapy, School of Medical Sciences, Tarbiat Modares University, Tehran, Iran.

2. Professor, Department of Physiotherapy, School of Medical Sciences, Tarbiat Modares University, Tehran, Iran.

3. Assistant Professor, Department of Physiotherapy, School of Medical Sciences, Tarbiat Modares University, Tehran, Iran

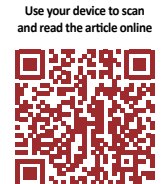

Citation: Khoshamooz Y, Torkaman G, Sarmadi A. The Recovery Curve of Soleus and Gastrocnemius H-Reflex After Applying Tripolar Electrical Stimulation on the Anesthetized Skin of Vertebral Column: An Emphasis on the Early and Secondary Facilitation Phases. Journal of Modern Rehabilitation. 2017; 11(1):3-12. https://doi.org/10.18869/nirp.jmr.11.1.3

https://doi.org/10.18869/nirp.jmr.11.1.3

Article info:

Received: 07 Aug. 2016

Accepted: 23 Nov. 2016
Keywords:

TENS, H-reflex, Local anesthesia, Motoneurons, Soleus muscle, Gastrocnemius muscle

\begin{abstract}
A B STRACT
Introduction: Computer modeling studies have shown that depolarization of the posterior and anterior root fibers can be achieved by surface tripolar electrical stimulation. This study investigated the soleus and gastrocnemius H-reflex recovery curve after applying tripolar electrical stimulation on the anesthetized skin of vertebral column. We emphasized on the early and secondary facilitation phases of the $\mathrm{H}$-reflex recovery curve.
\end{abstract}

Materials and Methods: A total of 15 healthy nonathletic female volunteers (mean $\pm \mathrm{SD}$ age: $27.20 \pm 3.17$ years) were enrolled in this study. H-reflex recovery curves of the soleus and gastrocnemius muscles were recorded before skin anesthesia and then 15 minutes after it (with lidocaine 10\%) and finally after application of Transcutaneous Electrical Nerve Stimulator (TENS) or placebo TENS (TENS on/off). Tripolar TENS with pulse width of 300 $\mu \mathrm{s}$, frequency of $80 \mathrm{~Hz}$ and intensity of 1.5 times of the sensory threshold was used for 15 minutes at the level of T11. To record the H-reflex recovery curve, 34 paired stimuli were used with the intensity of $30 \% \mathrm{H}$ max and interstimulus intervals between 1 and $202 \mathrm{~ms}$. After drawing the H-reflex recovery curve, amplitude, slope, area under the curve, and threshold of the early and secondary facilitation phases were calculated.

Results: After application of topical lidocaine, the sensory threshold of the skin vertebral column increased significantly. Fifteen minutes after applying local anesthesia, peak amplitude, slope, and area under the curve of the early facilitation phase significantly decreased in gastrocnemius and soleus muscles. After tripolar TENS application on the anesthetized skin of vertebral column, the slope of the early facilitation phase decreased significantly in the soleus $(\mathrm{P}=0.047)$. Also, area under the curve of the secondary facilitation phase significantly increased in both muscles.

Conclusion: These findings suggest the excitatory effect of skin anesthesia on the motoneurons activities. Changing the H-reflex recovery curve after application of tripolar TENS on the anesthetized skin may confirm deep penetration of this type of electrical stimulation.

* Corresponding Author:

Giti Torkaman, PhD

Address: Department of Physiotherapy, School of Medical Sciences, Tarbiat Modares University, Tehran, Iran.

Tel: +98 (21) 82884509

E-mail: torkamg@modares.ac.ir 


\section{Introduction}

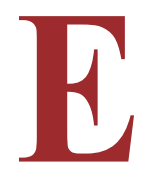

pidural electrical stimulation of the human spinal cord is a new and advanced therapeutic method introduced in 1967 [1], according to Melzack and Wall's theory [2]. Nowadays this method is used to control chronic pain [3-5], create changes in muscle tone and control spasticity $[6,7]$, and restore functional capacity in patients with spinal cord injury [8-10]. However, in many cases, the main goal is the stimulation of the spinal cord posterior column [11-13]. Recently many efforts have been done to stimulate the spinal structures with a simple non-invasive method using surface electrodes $[14,15]$.

Computer modeling studies have shown that depolarization of posterior and anterior root fibers can be achieved by bipolar surface electrical stimulation; but the stimulation intensity has to be increased at least three times. Moreover, raising the intensity will be intolerable for clinical applications [16, 17]. It is shown that tripolar electrical stimulation can affect the posterior column of the spinal cord [11, 18-21]. Some studies have been conducted to compare both bipolar and tripolar electrical stimulation on the motoneuron activity in spinal column area. Some inhibitory effects of motoneuron activity and H-response as well as reduction of spasticity, clonus, and flexion reflex were reported in previous studies [22-26].

The mechanism that is often expressed for explanation of these observations is inputs gain from skin receptors to motoneuron pool [22-26]. Goljaryan et al. evaluated the effects of vertebral column tripolar and bipolar stimulation on the H-reflex recruitment curve. They showed an inhibitory and an inhibitory-facilitory pattern in H-recruitment curve parameters after using bipolar and tripolar stimulation, respectively [25]. Navid et al. applied tripolar Transcutaneous Electrical Nerve Stimulator (TENS) on the anesthetized skin of vertebral column to investigate the role of skin receptors, in the transmission of electrical stimulation to the spinal cord [27]. Despite relative numbness in the skin, the excitability of the spinal cord motoneurons was shown after application of tripolar TENS [27].

In another study, Sarmadi et al. used tripolar TENS with various intensities on the vertebral column area and compared the soleus and gastrocnemius H-reflex recruitment curves. They evaluated the penetration depth of tripolar TENS and its effects on the slow and fast motoneurones. When the stimulus intensity was 1.5 times sensory threshold, facilitation of type II motor neurones and inhibition of type I motor neurons was seen. They stated that these responses may be due to transmission of tripolar TENS to dorsal horn. Tripolar TENS with lower intensity did not show such effects [28]. In all previous studies, more penetration depth of tripolar TENS, its transmission to posterior horn and progression to further stimulation of the Renshaw cells (with different impact on the type I and type II motoneurons) were suggested for different effects of tripolar and bipolar TENS [25, 27-30].

H-reflex recovery curve is a suitable and more accurate method for studying the behavior of motoneurons over time [31-34]. For recording, the pair sequential stimulation at different time intervals must be used. First stimulus is conditioning stimulus leading to $\mathrm{H} 1$ response and second stimulus is the test stimulus, with a defined interval from the first stimulus, leading to $\mathrm{H} 2$ response (test H-reflex). The interval between the pair stimulus, with equal intensity, should gradually increase motoneurons excitability until second stimulus be measured. Determination of $\mathrm{H} 2 / \mathrm{H} 1$ ratio in different intervals is used to obtain recovery curve. This method is a sensitive and accurate method to evaluate the changes in motor neurons activity in response to intervention in a specified time interval [35-37].

Applying the conditioning stimulus with a low intensity caused an early facilitation at the beginning of the recovery curve. This facilitation appears in the pair stimulation intervals of 3 to $15 \mathrm{~ms}$. The reason is the excitatory postsynaptic potential and facilitation of homonymous Ia fibers that its initial $3 \mathrm{~ms}$ is hidden by refractoriness of the axons which guided the flow [31-35, 38, 39]. After early facilitation, there is a strong inhibition (the period of primary unresponsiveness). At this point, $\mathrm{H} 2$ response cannot appear. This period is mainly due to recurrent inhibition of Renshaw cells and the phenomenon of after hyperpolarization $[39,40]$. The importance of early facilitation in this study is that Renshaw cells cannot participate at this period of the recovery curve and affect it [41-44].

In the present study, to clarify the role of Renshaw cells during tripolar TENS application, recovery curve of soleus and gastrocnemius muscles were recorded. Moreover, to determine the transmission of tripolar TENS through spinal posterior horn, the skin under electrodes was desensitized. With this method, skin receptors that are involved in changing the motoneurons activity will be numbness. Some evidence shows that the basic activity of skin receptors reduces the presynaptic inhibition of Ia afferents and therefore it has an excitatory effect on the motoneurons activity [27, 45-47]. Thus, desen- 
sitization of the skin by topical anesthetic agents may decrease the basic discharge of the low threshold skin receptors and cause inhibitory effects on the motorneurons excitability [27, 45-47].

However, there are some controversies that show the inhibitory effect of the basic discharge of the skin receptors on motoneurons activities [37, 48, 49]. Clinical application of tripolar TENS, regarding its better penetration, may be useful to change the muscle tone in different patients, but detailed studies are necessary to reveal its mechanisms. Cutaneous receptors are one of the important candidates to transmit the surface electrical stimulation to motoneurons level and subsequent inhibitory or facilitator processes on motor neurons, so in this study we attempted to investigate the soleus and gastrocnemius H-reflex recovery curve after applying tripolar electrical stimulation on the anesthetized skin of vertebral column. Obtained results can help us to clarify some details about tripolar TENS efficiency to change the slow and fast muscle activities.

\section{Materials and Methods}

\section{Design overview and study population}

This research was an experimental single-blind study, done in the laboratory of clinical electrophysiology of the Physical Therapy Department, Faculty of Medical Science, Tarbiat Modares University. Forty sedentary young women (mean \pm SD age: $27.20 \pm 3.17$ y) volunteered to participate in this study. Participants had normal body mass index (BMI, $18-25 \mathrm{~kg} / \mathrm{m}^{2}$ ) and no history of neurological, muscular, skeletal and articular diseases, fractures of the calf and ankle, deformities in the lower extremities, spine surgery, skin problems such as skin rashes, eczema or consumption of drugs, tobacco and alcohol. Participants were excluded from the study if H-reflex amplitude variability was more than $10 \%$ in five consecutive recording, co-appearance of H-reflex and $\mathrm{M}$ wave, and also when there was not early facilitation of H-reflex recovery curve.

Totally, 13 subjects because of inappropriate H-reflex, 9 subjects for missing the early facilitation phase of the H-reflex recovery curve, and 3 subjects for their personal reasons were excluded from this study. Finally, 15 participants were remained and examined in this study. This study was approved by the Ethics Committee of Tarbiat Modares University. Subjects were informed about the study design; each of them signed a written informed consent prior to participation.
Evaluation and treatment were done in three sessions for all subjects. In the first session, anthropometric variables, including age, height, weight, and body mass index of the participants were measured and calculated, then ankle dorsi and plantar flexion range of motion, patella and ankle reflexes, lower extremity muscle strength and tactile sensation of the skin for legs and vertebral column were evaluated. Five consecutive H-reflex and its recovery curve were measured to ensure of the $\mathrm{H}$ reflex variability of less than $10 \%$ and the existence of early facilitation in H-reflex recovery curve. Treatment was done in two separate sessions (skin anesthesia with TENS off and skin anesthesia with TENS on).

For all subjects, the second and third sessions took place at least 48 hours after the first and second sessions, respectively. H-reflex recovery curves of soleus and gastrocnemius muscles were recorded before intervention, 15 minutes after skin anesthesia (with lidocaine 10\%) [50], and finally after application of TENS on/off.

Sensory threshold of the electrical stimulation for the skin of vertebral column was evaluated. Skin temperature of vertebral column at the level of 12 thoracic vertebra and leg (overlying the soleus and gastrocnemius muscles on the right foot outward recorder electrodes) was measured by a digital thermometer (Iskra model 20) with an accuracy of 0.1 degrees. Then the accurate intensity to excite the gastrocnemius H-reflex with amplitude of 30\% Hmax was calculated. After initial recording of the soleus and gastrocnemius recovery curves, the skin of vertebral column (a rectangular region with a dimension of $24 \times 10$ $\mathrm{cm}$ centered to T11) was anesthetized. After ten minutes, the sensory threshold was measured again. Also, before and after anesthesia, the temperature of skin in the vertebral column area was measured. Then, 15 minutes of TENS of on or off was applied on the anesthetized skin of vertebral column. Immediately after applying TENS of on or off the recovery curves were recorded.

The recovery curves were recorded. Finally, skin temperature at the vertebral column and leg were measured. The participants in this study had no history of applying electrical stimulation or TENS. TENS current off and TENS current on were used in second and third sessions, respectively to prevent the subject's perception when the device is off. Room temperature was controlled between $23^{\circ} \mathrm{C}-27^{\circ} \mathrm{C}$ in all sessions.

\section{Recording the H-reflex}

Subjects were asked to lie prone with extended knee on the bed and freely moving of ankle joint from the 
bed edge. The skin under the electrodes was shaved and cleaned using alcohol to decrease the skin impedance. The H-reflex was recorded from the soleus and gastrocnemius muscles. To record the H-reflex, we used a computer-controlled stimulator with an isolator (Nihon Kohden ss-104j, Japan) and a two-channel Neuro-MEP system (Neurosoft, Russia). A round surface recording electrode $(\mathrm{Ag}-\mathrm{AgCl})$ with 1-cm diameter was used. The active recording electrode for soleus was placed on the middle of the virtual line between popliteal fossa to medial malleolus and the reference electrode $2 \mathrm{~cm}$ further distally [51].

The ground electrode was located between the stimulating and recording electrodes. The recording electrode for lateral gastrocnemius was placed around the upper 1/4 and the lower $3 / 4$ of the same virtual line and about 4 to 6 $\mathrm{cm}$ lateral to the midline of calf while positioned in parallel to the muscle fibers and 45 degrees with the midline of calf [52]. Bandwidth frequency and sampling frequency were set at $5 \mathrm{~Hz}, 10 \mathrm{kHz}$, and $20 \mathrm{kHz}$. The stimulating electrode was a bar electrode $3 \times 5.5 \mathrm{~cm}$ in size and with a $4 \mathrm{~cm}$ distance between cathode and anode. It was fixed in the popliteal fossa between biceps femoris and semimembranosus tendons, slightly toward the lateral. We used constant current stimulation, consisting of rectangular pulses with the pulse duration of $0.1 \mathrm{~ms}$ and a frequency of $0.2 \mathrm{~Hz}$, in order to prevent muscle fatigue.

\section{H-reflex recovery curve recording}

In order to record the H-reflex recovery curve, the sequenced paired stimuli with the same intensity, the accurate intensity to excite the soleus H-reflex with amplitude of $30 \% \mathrm{Hmax}$, were applied on the tibial nerve every $5 \mathrm{~s}$ (frequency of $0.2 \mathrm{~Hz}$ ). Initial interstimulus interval (ISI) was $1 \mathrm{~ms}$ that reached to $202 \mathrm{~ms}$ after 34 paired stimuli. Interstimulus interval for ten paired stimuli increased with the increments of $1 \mathrm{~ms}$ and for the others with the increments of $8 \mathrm{~ms}$. Because of the intrinsic variability of H-reflex amplitude, three recordings in each ISI were done and their mean was used for comparison.

\section{Drawing the H-reflex recovery curve}

Drawing H-reflex recovery curves was done by MATLAB. In each ISI, the mean peak to peak amplitude of $\mathrm{H} 1$ and $\mathrm{H} 2$ for three recordings and also $\mathrm{H} 2 / \mathrm{H} 1 \%$ were calculated and $\mathrm{H}$-reflex recovery curve of either soleus or gastrocnemius muscles were drawn for each participant (Figure 1). Peak amplitude, peak interval (interstimulus interval that peak was seen), area under the curve for the

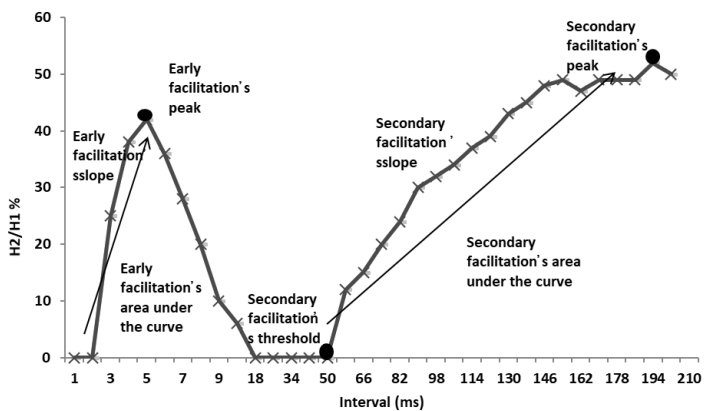

Figure 1. H-Reflex recovery curve

$J M R$

early facilitation phase, area under the curve and threshold of the secondary facilitation phase were calculated.

\section{TENS stimulus}

TENS stimulus was applied with a monophasic square pulse, frequency of $80 \mathrm{~Hz}$ and pulse width of $300 \mathrm{~ms}$ for 15 minutes. This stimulus was transferred by computer command to isolator (Nihon Kohden ss-104j, Japanse) with the ability of constant current, through an electronic intermediator. The electrical current of the isolator was manually adjustable from 0 to $30 \mathrm{~mA}$.

The cathode electrode was placed on the spinous process of T11 (existing point of S1 root from the intervertebral foramen). One anode electrode was placed 3 $\mathrm{cm}$ proximal and another one $3 \mathrm{~cm}$ distal to the cathode electrode. Rubber electrodes about $6 \times 4 \mathrm{~cm}^{2}$ were used. In order to apply the same pressure to the electrodes, we used a uniform taping methods; two adhesive strips diagonally and one strip horizontally were placed so that the entire surface of the electrode was in contact with the skin. The initial intensity of stimulation was set equal to 1.5 times the subject's sensory threshold intensity. To prevent the sensory adaptation, intensity increased 0.2 mA every 3 minutes. Maximum increment was $20 \%$ of initial intensity in each subject. In TENS off session, the electrodes were placed on the vertebral column at the same position, but the current was zero.

\section{Applying anesthetic and evaluation of sense of skin}

To apply the local anesthesia, $8 \mathrm{~mL}$ lidocaine $10 \%$ was dropped on the skin of vertebral column (a rectangular area of $24 \times 10 \mathrm{~cm}^{2}$ centered on T11) and massaged by the finger tips for 10 seconds. Before and after the skin anesthesia, sensory threshold was measured by TENS. The test was repeated three times within 10 seconds and the average value was considered as sensory threshold. 


\section{Data processing}

\section{Statistical analysis}

To analyze the data, SPSS 21 was used. To test the normal distribution of data, Kolmogorov-Smirnov test was used. We used ANOVA repeated measurement test to compare statistics in each session and paired $t$ test to compare the results in two sessions. The level of significance for all tests was set at $\alpha=0.05$.

\section{Results}

Anthropometric characteristics of participants are presented in Table 1. The sensory threshold of skin of vertebral column, showed a significant increase 15 minutes after topical anesthesia (Figure 2). Changes of the H-reflex recovery curve after application of lidocaine on the vertebral column skin in both sessions (Figures 3 and 4), 15 minutes after applying local anesthesia, peak amplitude , slope, and area under the curve of the primary facilitation phase significantly decreased in gastrocnemius and soleus muscles. Peak's interstimulus interval of the primary facilitation phase decreased in both muscles, but this diminution was not significant. Also threshold and its interstimulus interval of the second facilitation phase reduced significantly for both muscles.

The slope and area under the curve of the second facilitation phase showed an increase, but this increment was significant only for area under the curve. In the peak of both first and second phases of the recovery curve, after anesthesia, amplitude of $\mathrm{H} 1$ showed a significant increase in soleus and gastrocnemius muscle. Changes of the H-reflex recovery curve after application of tripolar TENS on the anesthetized skin of the vertebral column.

After 15 minutes of tripolar TENS application on the anesthetized skin of vertebral column, the slope of the early facilitation phase decreased significantly in the soleus muscle recovery curve. After TENS application, thresh- old and peak's interstimulus intervals of the second facilitation phase showed no significant increase in both soleus and gastrocnemius muscles. Area under the curve of the second facilitation phase significantly increased in both muscles (Figures 3 and 4). When sham TENS (TENS off) was used on the anesthetized skin, no significant changes were seen in the recovery curves parameters.

\section{Discussion}

The purpose of this study was to investigate the soleus and gastrocnemius H-reflex recovery curve after applying tripolar TENS on the anesthetized skin of vertebral column. Our results showed an increase in sensory threshold after application of topical anesthesia. This result confirms the effectiveness of lidocaine $10 \%$ to provide the anesthesia at the skin of vertebral column. After skin anesthesia, a significant reduction in the peak of the early facilitation phase was observed that might be due to a significant increase in the $\mathrm{H} 1$ amplitude. Also the threshold of the second facilitation phase decreased and its area under the curve increased significantly. These findings may confirm the excitatory effect of skin anesthesia on the motoneurons activity of the soleus and gastrocnemius muscles.

According to previous studies, local skin anesthesia causes an increase in the peak to peak amplitude of the $\mathrm{H}$ reflex. Persumably skin anesthesia may reduce the basal activity of the skin receptors and thereby decrease the presynaptic inhibition that increases the amplitude of the $\mathrm{H}$ reflex [37, 48, 49]. Basic activity of cutaneous receptors increases presynaptic inhibition of the Ia afferents [37, $48,49]$. Reduced activity of the skin receptors by local anesthesia or cooling leads to an increase in motor neuron excitability and increase in the amplitude of H-reflex through reducing the presynaptic inhibition $[37,53,54]$.

In the present study, this excitatory effect observed in the early facilitation phases of both soleus (as a slow twitch) and gastrocnemius (as a fast twitch) muscles. Ob-

Table 1. Anthropometric characteristics of participants

\begin{tabular}{cc}
\hline Variables & Mean \pm SD \\
\hline Age $(\mathrm{y})$ & $27.20 \pm 3.17$ \\
Height $(\mathrm{cm})$ & $1.6 \pm 0.08$ \\
Weight $(\mathrm{kg})$ & $53.32 \pm 6.81$ \\
\hline Body mass index $\left(\mathrm{kg} / \mathrm{m}^{2}\right)$ & $20.81 \pm 2.09$ \\
\hline
\end{tabular}




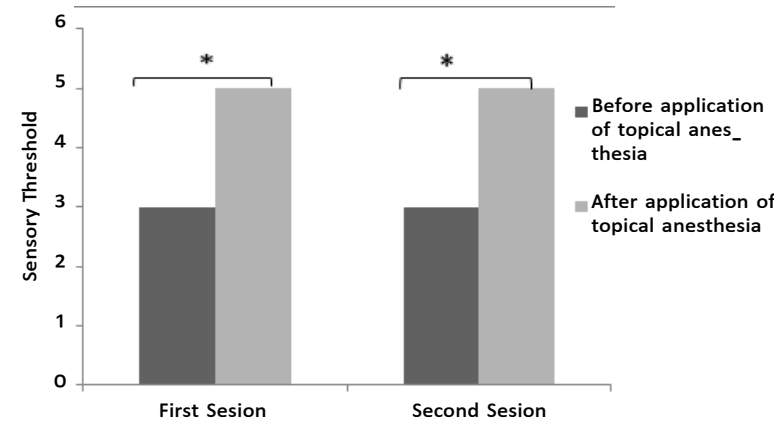

$J \mathrm{MR}$

Figure 2. Changes of the sensory threshold after application of topical anesthesia

viously, the strongest inhibitory effect of Renshow cells is on the slow motoneurons [34, 40], but the Renshaw cell activity cannot be seen during the early facilitation phase (the interstimulus interval less than $10 \mathrm{~ms}$ ), so a similar facilitation response was seen in both muscles.

In a study conducted by Sabbahi et al. after applying the local anesthesia on the different skin areas and dermatomes peak to peak amplitude of the H-reflex increased significantly, except when anesthesia was done on the S1 dermatome and on the skin covering the tibialis anterior muscle [37]. Also in our study, a significant reduction was observed in the threshold of the second facilitation phase, the peak's interstimulus interval and an increase in the area under the curve. The second facilitation phase of the H-reflex recovery curve is an indirect measurement of the suprasegmental control over the spinal cord motor neurons [46].

Thus, the facilitating effects of local anesthetic on the second facilitation phase can also be attributed to supraspinal effects and long loop inputs. Lewis and Porter showed the pyramidal neurons discharge increase after blocking inputs of the skin afferent by local anesthesia. In this study, H-reflex recovery curve facilitation was observed in shorter intervals (less than $10 \mathrm{~ms}$ ), which may represent the segmental effects. Decrease in the threshold of the second facilitation phase after applying local skin anesthesia can be attributed to the reduction of Renshaw cell activity [55].

Applying sham TENS (TENS off) on the desensitized skin caused no change in the recovery curve parameters; this reflects the long lasting effects of sensory impairment in the skin and reduction of basic activity of cutaneous receptors. Applying tripolar TENS on the desensitized skin caused a significant reduction in the slope of the early facilitation phase in the soleus muscle. As previously mentioned, Renshaw cells cannot act during

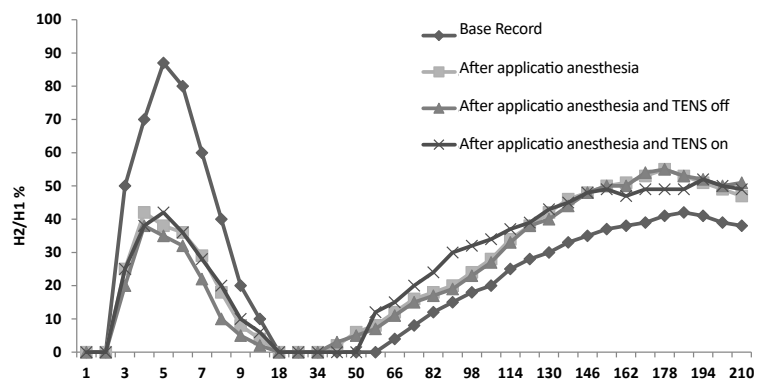

$J \mathrm{MR}$

Figure 3. H-reflex recovery curves of the gastrocnemius muscle before and after application of anesthesia, anesthesia with TENS off, and anesthesia with TENS on

the early facilitation period and especially in the interstimulus interval under $10 \mathrm{~ms}$, therefore probably this inhibitory effect was caused by the current transmission of tripolar TENS through the dorsal horn. Tripolar TENS stimulates the skin afferents, especially the group II and III afferents (low threshold receptors), and also the dorsal horn of the spinal cord $[25,27,28,30]$.

The effect of skin afferents on the type I motoneurons is inhibitory and on the type II motoneurons is facilaitory $[25,27,28,30]$. Thus, skin efferent excitation leads to type II motor neurons facilitation and type I motoneurons inhibition $[28,30]$. Increasing the activity of type II motoneurons leads to an increase in the Renshaw cell activity and subsequently more inhibition of type I motoneurons $[28,30]$. Tripolar TENS with more penetration depth, probably affects dorsal horn of the spinal cord and stimulates Ia and Ib afferents. Ia afferent stimulates both types of motoneurons but has more facilitatory effect on type I motor neurons. The effect of Ib fibers on the type I motoneurons is inhibitory and on the type II motoneurons is facilitatory $[25,27,28,30]$.

Given the role of Renshaw cells in the early facilitation period is ruled out, therefore soleus muscle inhibition may be due to the excitation of $\mathrm{Ib}$ fibers in the

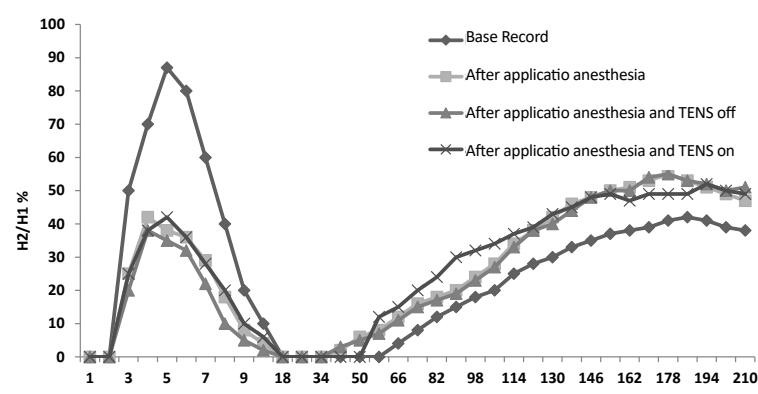

Figure 4. H-reflex recovery curves of the soleus muscle before and after application of anesthesia, anesthesia with TENS off, and anesthesia with TENS on 
dorsal horn by deep penetration of tripolar TENS. The observed increase in the threshold and interstimulus interval of peak in the second facilitation phase, in both muscles may indicate an increase in reactivation of the Renshaw cells. However, because of the Renshaw's stronger effect on slow-twitch motoneurons, it was expected that this inhibitory effect was more prominent in soleus than gastrocnemius muscle, but we recorded the recovery curve with the intensity of $30 \% \mathrm{Hmax}$. In this intensity, mainly low threshold fibers (Type I) in both muscles were stimulated.

To stimulate the fast-twitch fibers (Type II) through the $\mathrm{H}$-reflex path, higher stimulation intensity is needed. It is shown that to stimulate the muscle II fibers, we need an intensity of 2-5 times greater than the stimulation threshold of type I fibers [30]. Simorgh et al. observed that after applying tripolar TENS, the amplitude of the maximum recovery increased in the gastrocnemius muscle and in a shorter interstimulus interval, whereas the amplitude of the maximum recovery in soleus muscle decreased and obtained in a longer interstimulus interval. Soleus and gastrocnemius H-reflex recovery curves in their study were recorded in interstimulus interval of 40-520 ms and intensity of $70 \% \mathrm{Hmax}[30]$.

Sarmadi et al. also showed that applying tripolar TENS with the intensity of 1.5 times the sensory threshold had a complex facilitatory-inhibitory effect on the recruitment curve of H-reflex. They observed slow motoneurons inhibition and fast motoneurons facilitation. In the gastrocnemius muscle, the ascending slope of the recruitment curve increased and the needed intensity to evoke Hmax decreased [28].

A recent study that examined the cathodal and anodal stimulation at the level of T11 on the motoneuron excitability showed that the surface vertebral column stimulation can alter the spinal cord conductive properties and affect the evoked potentials $[26,56,57]$. Cogiamanian et al. showed that transcutaneous anodal direct current decreased significantly the amplitude of the cervicomedullary component of somatosensory evoked potential of the posterior tibial nerve about $25 \%$. On the contary, cathodal current leads to increase the amplitude of this component [56]. Winkler et al. showed applying anodal current induced significantly decrease in H-reflex post activation depression while cathodal current induced significantly increase in the post activation depression. They showed no significant change in the ratio of Hmax/Mmax [26].

Lamy and colleagues investigated the effect of surface stimulation of vertebral column, at the T11 level, on the soleus H-reflex recruitment curve. They showed anodal stimulation induced a progressive leftward shift of the $\mathrm{H}$ reflex recruitment curve and increased excitability of motor neurons. In contrast, cathodal stimulation had no significant effects. They suggested this increament was due to decrease inthe post-activation depression [57]

The results showed that the relative decrease in activity of the skin receptors after local anesthesia, through reducing baseline activity of the skin receptors and subsequently increase of the presynaptic inhibition in Ia afferent, can increase excitability of motoneurons and facilitated the H-reflex recovery curve in both soleus and gastrocnemius muscles.

After applying tripolar TENS, an inhibitory response was seen at the early facilitation phase of the soleus recovery curve. This inhibition may be due to the excitation of Ib fibers in the dorsal horn by deep penetration of tripolar TENS, because there is no action of the renshaw cells at interstimulus interval less than $10 \mathrm{~ms}$ (appearance time of the early facilitation phase). Moreover, the threshold increase in the second facilitation phase in both muscles may be attributed to the recurrent inhibition. Probably, deep penetration of tripolar TENS, despite the skin anesthesia, has excited Ia and Ib fibers and subsequently Renshaw cells with their inhibitory effects on the spinal motoneurons.

\section{Clinical application of tripolar TENS}

Tripolar TENS because of its deep penetration may be a useful modality to change the spinal motoneuron activity. In some patients, we need to improve the abnormal muscle tone. It may be possible through using the different types of electrical stimulation. The effect of tripolar TENS on the improvement of muscle weakness and also excitation or inhibition of the motoneuron activities, in some pathophysiologic conditions, should be considered in future studies.

\section{Acknowledgments}

This study was accomplished in clinical electrophysiology lab of Physical Therapy Department and was funded by a grant from the Postgraduate Studies and Research Program at Medical Science faculty, Tarbiat Modares University, Tehran, Iran

\section{Conflict of Interest}

The authors declared no conflicts of interest. 


\section{References}

[1] Shealy CN, Mortimer JT, Reswick JB. Electrical inhibition of pain by stimulation of the dorsal columns. Anesthesia \& Analgesia. 1967; 46(4):489-91. doi: 10.1213/00000539-19670700000025

[2] Melzack R, Wall PD. Pain mechanisms: A new theory. Science. 1965; 150(3699):971-8. doi: 10.1126/science.150.3699.971

[3] Hegarty D, Goroszeniuk T. Peripheral nerve stimulation of the thoracic paravertebral plexus for chronic neuropathic pain. Pain Physician. 2011; 14(3):295-300. PMID: 21587333

[4] North RB, Kidd DH, Farrokhi F, Piantadosi SA. Spinal cord stimulation versus repeated lumbosacral spine surgery for chronic pain: A randomized, controlled trial. Neurosurgery. 2005; 56(1):98-107. doi: 10.1227/01.neu.0000144839.65524.e0

[5] Shrivastav M, Musley S. Spinal cord stimulation for complex regional pain syndrome. Paper presented at the 2009 $31^{\text {st }}$ Annual International Conference of the IEEE Engineering in Medicine and Biology Society. 3-6 September 2009; Minneapolis, Minnesota, United States. doi: 10.1109/ iembs.2009.5334418

[6] Dimitrijevic MR, Illis LS, Nakajima K, Sharkey PC, Sherwood AM. Spinal cord stimulation for the control of spasticity in patients with chronic spinal cord injury: II. Neurophysiologic observations. Central Nervous System Trauma. 1986; 3(2):145-52. doi: 10.1089/cns.1986.3.145

[7] Pinter MM, Gerstenbrand F, Dimitrijevic MR. Epidural electrical stimulation of posterior structures of the human lumbosacral cord: 3. Control of spasticity. Spinal Cord. 1999; 38(9):524-31. Doi: 10.1038/sj.sc.3101040

[8] Minassian K, Jilge B, Rattay F, Pinter MM, Binder H, Gerstenbrand F, et al. Stepping-like movements in humans with complete spinal cord injury induced by epidural stimulation of the lumbar cord: Electromyographic study of compound muscle action potentials. Spinal Cord. 2004; 42(7):401-16. doi: 10.1038/sj.sc.3101615

[9] Minassian K, Persy I, Rattay F, Pinter MM, Kern H, Dimitrijevic MR. Human lumbar cord circuitries can be activated by extrinsic tonic input to generate locomotor like activity. Human Movement Science. 2007; 26(2):275-95. doi: 10.1016/j. humov.2007.01.005

[10] Harkema S, Gerasimenko Y, Hodes J, Burdick J, Angeli C, Chen $Y$, et al. Effect of epidural stimulation of the lumbosacral spinal cord on voluntary movement, standing, and assisted stepping after motor complete paraplegia: A case study The Lancet. 2011; 377(9781):1938-47. doi: 10.1016/s01406736(11)60547-3

[11] Holsheimer J, Struijk JJ, Tas NR. Effects of electrode geometry and combination on nerve fibre selectivity in spinal cord stimulation. Medical \& Biological Engineering \& Computing. 1995; 33(5):676-82. doi: 10.1007/bf02510785

[12] Wesselink WA, Holsheimer J, Nuttin B, Boom HBK, King GW, Gybels JM, et al. Estimation of fiber diameters in the spinal dorsal columns from clinical data. IEEE Transactions on Biomedical Engineering. 1998; 45(11):1355-62. doi: $10.1109 / 10.725332$

[13] Feirabend HKP, Choufoer H, Ploeger S, Holsheimer J, van Gool JD. Morphometry of human superficial dorsal and dor- solateral column fibres: Significance to spinal cord stimulation. Brain. 2002; 125(5):1137-49. doi: 10.1093/brain/awf111

[14] Minassian K, Persy I, Rattay F, Dimitrijevic MR, Hofer C, Kern $\mathrm{H}$. Posterior root muscle reflexes elicited by transcutaneous stimulation of the human lumbosacral cord. Muscle \& Nerve. 2007; 35(3):327-36. doi: 10.1002/mus.20700

[15] Hofstoetter US, Minassian K, Hofer C, Mayr W, Rattay F, Dimitrijevic MR. Modification of reflex responses to lumbar posterior root stimulation by motor tasks in healthy subjects. Artificial Organs. 2008; 32(8):644-8. doi: 10.1111/j.15251594.2008.00616.x

[16] Ladenbauer J, Minassian K, Hofstoetter US, Dimitrijevic MR, Rattay F. Stimulation of the human lumbar spinal cord with implanted and surface electrodes: A computer simulation study. IEEE Transactions on Neural Systems and Rehabilitation Engineering. 2010; 18(6):637-45. doi: 10.1109/ tnsre.2010.2054112

[17] Danner SM, Hofstoetter US, Ladenbauer J, Rattay F, Minassian $\mathrm{K}$. Can the human lumbar posterior columns be stimulated by transcutaneous spinal cord stimulation? A modeling study. Artificial Organs. 2011; 35(3):257-62. doi: 10.1111/j.1525-1594.2011.01213.x

[18] Struijk JJ, Holsheimer J, Spincemaille GHJ, Gielen FLH Hoekema R. Theoretical performance and clinical evaluation of transverse tripolar spinal cord stimulation. IEEE Transactions on Rehabilitation Engineering. 1998; 6(3):277-85. doi: $10.1109 / 86.712224$

[19] Holsheimer J, Struijk JJ. Electrode geometry and preferential stimulation of spinal nerve fibers having different orientations: A modeling study. Paper presented at the $199214^{\text {th }}$ Annual International Conference of the IEEE Engineering in Medicine and Biology Society. 29 October-1 November 1992; Paris, France. doi: 10.1109/iembs.1992.5761836

[20] Deurloo KEI, Holsheimer J. Transverse tripolar stimulation for selective FNS. Paper presented at the $18^{\text {th }}$ Annual International Conference of the IEEE Engineering in Medicine and Biology Society. 31 October-3 November 1996; Amsterdam, The Netherlands. doi: 10.1109/iembs.1996.656978

[21] Holsheimer J, Wesselink WA. Optimum electrode geometry for spinal cord stimulation: The narrow bipole and tripole. Medical \& Biological Engineering \& Computing. 1997; 35(5):493-7. doi: 10.1007/bf02525529

[22] Wang RY, Tsai MW, Chan RC. Effect of surface spinal cord stimulation on spasticity and quantitative assessment of muscle tone in hemipelgic patient. American Journal of Physical Medicine \& Rehabilitation. 1998; 77(4):282-7. doi 10.1097/00002060-199807000-00003

[23] Ray Yau W, Rai Chi C, Tsai MW. Effects of thoraco lumbar electric sensory stimulation on knee extensor spasticity of persons who survived cerebrovascular accident (CVA). Journal of rehabilitation research and development. 2000; 37(1):73-80

[24] Hiraoka K. Neural mechanisms underlying the effect of transcutaneous electrical nerve stimulation in humans. Electromyography and Clinical Neurophysiology. 2002; 42(6):359 66. PMID: 12224473

[25] Goljaryan S, Firoozabadi SMP, Torkaman G. The effects of bi-polar and tri-polar TENS on H-reflex and Mh wave recruitment curve. Paper presented at the $2^{\text {nd }}$ Europian Medical 
and Biological Engineering Conference. 4-8 December 2002; Vienna, Austria.

[26] Winkler T, Hering P, Straube A. Spinal DC stimulation in humans modulates post activation depression of the H-reflex depending on current polarity. Clinical Neurophysiology. 2010; 121(6):957-61. doi: 10.1016/j.clinph.2010.01.014

[27] Navid A, Torkamani G, Firoozabadi SM. [Evaluation of H-reflex recruitment curve after application of TENS on the desensitised skin of vertebral column (Persian)]. Physiology and Pharmacology. 2003; 7(2):123-33.

[28] Sarmadi A, Torkaman G. [The effects of vertebral column tripolar electrical stimulation with various intensities on soleus and gastrocnemius H-reflex and $\mathrm{Mh}$ wave recruitment curve (Persian)]. Physiology and Pharmacology. 2009; 13(2):229-43.

[29] Saeedi N, Firoozabadi SMP, Torkaman G, Kazemnejad A The effect of tripolar percutaneous Lumbar stimulation on the H-reflex recruitment curves. Paper presented at the $3^{\text {rd }}$ International Conference on Business Management and Economics. 2 December 2016; Colombo, Srilanka.

[30] Simorgh L, Torkaman G, Firoozabadi SM. [Effects of tripolar TENS of vertebral column on slow and fast motor units: A preliminary study using H-reflex recovery curve method (Persian)]. Physiology and Pharmacology. 2007; 10(4):231-42.

[31] Magladery JW, Teasdall RD, Park AM, Languth HW. Electrophysiological studies of reflex activity in patients with lesions of the nervous system; A comparison of spinal motoneurone excitability following afferent nerve volleys in normal persons and patients with upper motor neurone lesions. Bulletin of the Johns Hopkins Hospital. 1952; 91(4):219-44. PMID: 12987919

[32] Táboříková H, Sax DS. Conditioning of H-reflexes by a preceding subthreshold H-reflex stimulus. Brain. 1969; 92(1):20312. doi: $10.1093 /$ brain/92.1.203

[33] Gassel MM. A critical review of evidence concerning long loop reflexes excited by muscle afferents in man. Journal of Neurology, Neurosurgery \& Psychiatry. 1970; 33(3):358-62. doi: 10.1136/jnnp.33.3.358

[34] Fukushima YA, Yamashita NA, Shimada Y. Facilitation of H-reflex by homonymous Ia-afferent fibers in man. Journal of neurophysiology. 1982; 48(5):1079-88.

[35] Kagamihara Y, Hayashi A, Okuma Y, Nagaoka M, Nakajima Y, Tanaka R. Reassessment of H-reflex recovery curve using the double stimulation procedure. Muscle \& Nerve. 1998; 21(3):352-60.

[36] Sabbahi M, Etnyre B, Al Jawayed IA, Hasson S, Jankovic J. Methods of H-reflex evaluation in the early stages of Parkinson's disease. Journal of Clinical Neurophysiology. 2002; 19(1):67-72. doi: 10.1097/00004691-200201000-00009

[37] Sabbahi M, De Luca C. Topical anesthesia: H-reflex recovery changes by desensitization of the skin. Electroencephalography and Clinical Neurophysiology. 1981; 52(4):328-35. doi: 10.1016/0013-4694(81)90061-4

[38] Magladery JW, Porter WE, Park AM, Teasdall RD. Electrophysiological studies of nerve and reflex activity in normal man; IV. The two neurone reflex and identification of certain action potentials from spinal roots and cord. Bulletin of the Johns Hopkins Hospital. 1951; 88(6):499-519.
[39] Pierrot Deseilligny E. Evidence for supraspinal influences on renshaw inhibition during motor activity in man. Progress in Clinical Neurophysiology. 1980; 8:142-69.

[40] Pierrot Deseilligny E, Bussel B, Held J., Katz R. Excitability of human motoneurones after discharge in a conditioning reflex. Electroencephalography and Clinical Neurophysiology. 1976; 40(3):279-87. doi: 10.1016/0013-4694(76)90151-6

[41] Veale JL, Rees S. Renshaw cell activity in man. Journal of Neurology, Neurosurgery \& Psychiatry. 1973; 36(4):674-83. doi: 10.1136/jnnp.36.4.674

[42] McLeod JG. H-reflex studies in patients with cerebellar disorders. Journal of Neurology, Neurosurgery \& Psychiatry. 1969; 32(1):21-7. doi: 10.1136/jnnp.32.1.21

[43] Olsen PZ, Diamantopoulos E. Excitability of spinal motor neurones in normal subjects and patients with spasticity, Parkinsonian rigidity, and cerebellar hypotonia. Journal of Neurology, Neurosurgery \& Psychiatry. 1967; 30(4):325-31. doi: 10.1136/jnnp.30.4.325

[44] McLeod JG, Walsh JC. H reflex studies in patients with Parkinsons disease. Journal of Neurology, Neurosurgery \& Psychiatry. 1972; 35(1):77-80. doi: 10.1136/jnnp.35.1.77

[45] Bagheri R, Sarmadi A, Torkaman G. [Effects of the lower leg tapping on $\mathrm{H}$ reflexes recruitment curve parameters: a comparison of Soleus and lateral Gastrocnemius muscles motor neurons (Persian)]. Journal of Shahrekord University of Medical Sciences. 2012; 14(4):1-0

[46] Agostinucci J. The effect of topical anesthetics on skin sensation and soleus motoneuron reflex excitability. Archives of Physical Medicine and Rehabilitation. 1994; 75(11):1233-40. doi: 10.1016/0003-9993(94)90011-6

[47] Salehi H, Torkaman G, Firoozabadi SM. [The effect of spinal skin cooling with topical ointment on the recruitment curve parameters of soleus H-reflex (Persian)]. Physiology and Pharmacology. 2006; 10(2):151-7.

[48] Sabbahi M., De Luca C. Topical anesthesia: Modulation of the monosynaptic reflexes by desensitization of the skin. Electroencephalography and Clinical Neurophysiology. 1982; 54(6):677-88. doi: 10.1016/0013-4694(82)90122-5

[49] Sabbahi MA, De Luca CJ, Powers WR. Topical anesthesia: A possible treatment method for spasticity. Archives of Physical Medicine and Rehabilitation. 1981; 62(7):310-4. PMID: 7247657

[50] Khoshamooz Y, Torkaman G, Sarmadi A. [The anesthesia effect of vertebral column skin receptors on the motoneuron excitability and H-reflex recovery in Soleus and Gastrocnemius muscles (Persian)]. Journal of Modern Rehabilitation. 2015; 9(4):54-67.

[51] Kimura J. Electrodiagnosis in diseases of nerve and muscle: Principles and practice. Oxford: Oxford University Press; 2013. doi: $10.1093 /$ med/9780199738687.001.0001

[52] Sarmadi A, Firoozabadi SM, Torkaman G, Fathollahi Y. Assessing information of soleous and gastrocnemius motor unit H-reflex response to paired stimulation. Electromyography and Clinical Neurophysiology. 2003; 44(7):401-8.

[53] Martins FL, Carvalho LC, Silva CC, Brasileiro JS, Souza TO, Lindquist ARR. Immediate effects of TENS and cryotherapy in the reflex excitability and voluntary activity in hemiparetic 
subjects: A randomized crossover trial. Brazilian Journal of Physical Therapy. 2012; 16(4):337-44. doi: 10.1590/s141335552012005000032

[54] Allison SC, Abraham LD. Sensitivity of qualitative and quantitative spasticity measures to clinical treatment with cryotherapy. International Journal of Rehabilitation Research. 2001; 24(1):15-24. doi: 10.1097/00004356-200103000-00003

[55] Patton HD. Textbook of physiology: Excitable cells and neurophysiology. Philadelphia: WB Saunders Company; 1989.

[56] Cogiamanian F, Vergari M, Pulecchi F, Marceglia S, Priori A. Effect of spinal transcutaneous direct current stimulation on somatosensory evoked potentials in humans. Clinical Neurophysiology. 2008; 119(11):2636-40. doi: 10.1016/j. clinph.2008.07.249

[57] Lamy JC, Ho C, Badel A, Arrigo RT, Boakye M. Modulation of soleus H-reflex by spinal DC stimulation in humans. Journal of Neurophysiology. American Physiological Society. 2012; 108(3):906-14. doi: 10.1152/jn.10898.2011 